\title{
Study on the Phase Transition Behavior of Polymerized Liposomes through the Interaction of Diene-Groups in Their Acyl Chains
}

\author{
Shinji TAKeOKa, Hiromi SAKaI, Ling WaNG, \\ Hiroyuki OHNO, ${ }^{\dagger}$ and Eishun TsUCHIDA* \\ Department of Polymer Chemistry, Waseda University, \\ Tokyo 169, Japan
}

(Received March 6, 1989)

\begin{abstract}
Bis(2,4-octadecadienoyl)-sn-glycero-3-phosphorylcholine (DODPC), which had a diene group in 2,4-position of 1- and 2-acyl chains, was used to detect phase transition from shift of absorption maximum $\left(\lambda_{\max }\right)$ due to the change of interaction of diene groups. Selective polymerization of 1-acyl chains of DODPC with AIBN gave linear phospholipid polymers which had unreacted diene groups in 2-acyl chains. From the analysis of $\lambda_{\max }$ of thus selectivelypolymerized liposomes, the phase transition behavior was detected clearly. We also studied the phase transition behavior of liposomes of which either layer of bilayer was selectively polymerized. Reconstitution of polymerized DODPC liposomes could also be evaluated from the temperature dependence of $i_{\text {max }}$. The molecular packing of the polymeric liposomes was disturbed by reconstitution because of lower freedom of phospholipid polymers on reconstitution process.
\end{abstract}

KEY WORDS Liposome / Phase Transition / Polymerized Liposomes /

Reconstitution / Diene-Containing Lipids / Absorption Maximum /

The liposome has hitherto been studied for the application of drug delivery system because both hydrophobic bilayer and an inner aqueous phase can be used for encapsulation of functional molecules. ${ }^{1.2}$ Liposomes, which can release entrapped materials in response to the outer stimuli, were designed and studied in physiological conditions. ${ }^{2}$ Liposomes tend to aggregate and fuse after storage at low temperature. $^{3}$ They are not stable enough and sometimes release encapsulated molecules easily in vivo or in vitro. ${ }^{2}$ Polymerization of liposomes components is a potent technique to prepare the stable liposomes. We suicceeded in preparing polymerized mixed liposomes which released encapsulated molecules in response to physical or chemical stimuli. ${ }^{4} \mathrm{CF}$ release measurement, electron microscopy and light-scat- tering measurement were often applied to analyze the stability of polymerized liposomes. ${ }^{5-7}$ There were a few methods to evaluate phase transition behavior of polymerized liposome membrane such as photobleaching ${ }^{8}$ and DSC measurement. ${ }^{9}$

We have already used amphiphilic porphynato zinc, 3,8,13,17-tetramethyl-7,12-dicarboxy-2,18-bis(octadecylpropinate)porphynato zinc (DCPZn) as a membrane probe for the detection of its phase transition temperature. ${ }^{10}$ The fluorescence intensity changed in relation to the change of dissociation-association equilibrium of the amphiphilic porphyrins at temperatures below and above the phase transition temperature. It was however impossible to detect the phase transition of polymerized liposomes with this method, because these

\footnotetext{
* To whom all correspondence should be addressed.

+ Present address; Faculty of Technology, Tokyo University of Agriculture and Technology, Tokyo 184.
} 
probe molecules were phase separated and fixed by the polymerized lipids.

On the other hand, we studied the polymerization behavior of 1,2-bis(2,4-octadecadienoyl)-sn-glycero-3-phosphorylcholine (DODPC) as liposomes. ${ }^{11-16}$ We succeeded in conducting the selective polymerization of diene groups in 1- or 2-acyl chain by the use of radical initiators having different solubility. ${ }^{11}$ Since diene-groups of 1-acyl chains were located in hydrophobic region of bilayer, they were selectively polymerized with water insoluble azobis(isobutyronitrile) (AIBN). While, those of 2-acyl chains were polymerized with watersoluble azobis(2-amidinopropane) dihydrochloride (AAPD) because they faced an aqueous phase. ${ }^{11}$ Furthermore, we found that the absorption maximum $\left(\lambda_{\max }\right)$ of diene groups in DODPC shifted to shorter wavelengths when the assembly state of DODPC liposomes changed from liquid-crystalline to gel state. ${ }^{17}$ Since the behavior was explained in terms of the interaction of diene groups, the $\lambda_{\max }$ was also influenced by molecular packing and the orientation of diene groups. DODPC liposomes were polymerized with AIBN to obtain linear phospholipid polymers which had unreacted diene groups in only 2-acyl chains. The $\lambda_{\max }$ shift of diene groups in the polymers should also reflect the phase transition of the polymerized liposomes.

In this paper, we analyzed the polymerized liposomes through the spectral shift after introducing probe moieties (diene groups) directly to the 2,4-position of acyl chains of phospholipid polymers.

\section{EXPERIMENTAL}

\section{Materials}

1,2-Bis(2,4-octadecadienoyl)-sn-glycero-3phosphorylcholine (DODPC) containing 2,4diene group in both 1- and 2-acyl chain was purchased from Nippon Oil \& Fats Co., Ltd. The purity of the lipid was confirmed with thin layer chromatography (Merck, silica gel plates) with chloroform-methanol-water (65: $35: 5$ by vol). Samples showing single spot with an $R f$ value of around 0.3 were used for experiments. Azobis(isobutyronitrile) (AIBN) was purchased from Tokyo Kasei Co., Ltd. and was purified twice by recrystallization from methanol.

\section{1-(2,4-Octadecadienoyl )-2-hydroxy-sn-gly- cero-3-phosphorylcholine}

1,2-Bis(2,4-octadecadienoyl)-sn-glycero-3phosphorylcholine (DODPC) $(10.0 \mathrm{~g}, 12.8 \mathrm{mM})$ was dissolved in dichloromethane. After the addition of $200 \mathrm{ml}$ of $0.2 \mathrm{M}$ Tris buffer $(\mathrm{pH}$ 8.14 ), dichloromethane was completely removed by $\mathrm{N}_{2}$ flow at $30^{\circ} \mathrm{C}$. The resulting multilamellar DODPC liposomes were extruded sequentially through polycarbonate filters $(0.6$ $\mu \mathrm{m}, 0.2 \mu \mathrm{m}$, and then $0.1 \mu \mathrm{m})$ to convert them to small unilamellar liposomes. Phospholipase $\mathrm{A}_{2}(0.20 \mathrm{~g})$ and $\mathrm{CaCl}_{2} \cdot \mathrm{H}_{2} \mathrm{O}(0.375 \mathrm{~g})$ were added to the liposome suspension. The mixture was reacted for $20 \mathrm{~h}$ at $35^{\circ} \mathrm{C}$ in dark. After the extraction of the lyophilized powder with chloroform, the extract was separated by a column chromatography on silica gel with $\mathrm{CHCl}_{3}-\mathrm{MeOH}-\mathrm{H}_{2} \mathrm{O}(65: 25: 4$ by vol). The fraction $(R f=0.17)$ were collected and freezedried from benzene to obtain 1-(2,4-octadecadienoyl)-2-hydroxy-sn-glycero-3-phosphorylcholine $(5.40 \mathrm{~g}$, yield $81 \%)$. IR spectrum (in

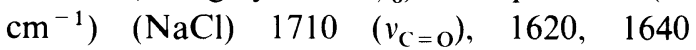
$\left(v_{\mathrm{C}=\mathrm{C}}\right), \quad 1240 \quad\left(v_{\mathrm{P}=\mathrm{O}}\right), \quad 1090 \quad\left(v_{\mathrm{P}-\mathrm{O}-}\right), \quad 1060$ $\left(\delta_{\mathrm{P}-\mathrm{O}-\mathrm{C}}\right)$; UV spectrum, $264.9 \mathrm{~nm}$ (in $\left.\mathrm{MeOH}\right)$ $(\mathrm{C}=\mathrm{C}-\mathrm{C}=\mathrm{C}-\mathrm{C}(\mathrm{O})-),{ }^{1} \mathrm{H}$ NMR $\left(\mathrm{CD}_{3} \mathrm{OD}\right.$, TMS) (in ppm): $0.88\left(3 \mathrm{H}, \mathrm{CH}_{3} \mathrm{CH}_{2}-\right), 1.28$ $\left(22 \mathrm{H}, \mathrm{CH}_{2}\right), 2.20\left(2 \mathrm{H}, \mathrm{CH}_{2} \mathrm{CH}=\right), 3.35(9 \mathrm{H}$, $\left.-\mathrm{N}^{+} \mathrm{CH}_{3}\right), 5.74-7.45(4 \mathrm{H}$, diene).

\section{1-(2,4-Octadecadienoyl)-2-palmitoyl-sn-gly- cero-3-phosphorylcholine}

1-(2,4-Octadecadienoyl)-2-hydroxy-sn-glycero-3-phosphorylcholine $(3.50 \mathrm{~g}, 6.7 \mathrm{mM})$ was dissolved in $100 \mathrm{ml}$ of dry dichloromethane. 4Dimethyl aminopyridine $(8.20 \mathrm{~g}, 67 \mathrm{mM})$ and palmitoylchloride $(18.4 \mathrm{~g}, 67 \mathrm{mM})$ were slowly 
added to the solution at $0^{\circ} \mathrm{C}$. The mixture was stirred at room temperature in the dark under nitrogen atmosphere for three days. The solution was washed with excess water and evaporated to dryness under reduced pressure. The residue was dried in vacuo over $\mathrm{P}_{2} \mathrm{O}_{5}$. The residue was washed a few times with acetone. Purification was carried out by passing specify through a silica gel column with $\mathrm{CHCl}_{3}-\mathrm{MeOH}-\mathrm{H}_{2} \mathrm{O}$ (65:25:4 by vol). The fractions $(R f=0.5)$ were collected. The lyophilization from benzene afforded 1-(2,4-octadecadienoyl)-2-palmitoyl-sn-glycero-3-phosphorylcholine $(0.80 \mathrm{~g}, 11 \mathrm{mM}$, yield $15 \%)$. IR spectrum (in $\left.\mathrm{cm}^{-1}\right)(\mathrm{NaCl}) 1710\left(v_{\mathrm{C}=\mathrm{C}}\right), 1240$ $\left(v_{\mathrm{P}=\mathrm{o}}\right), 1090\left(v_{\mathrm{P}-\mathrm{O}-}\right), 1060\left(\delta_{\mathrm{P}-\mathrm{O}-\mathrm{C}}\right) ; \mathrm{UV}$ spectrum, $264.9 \mathrm{~nm}$ (in $\left.\mathrm{CHCl}_{3}\right)(\mathrm{C}=\mathrm{C}-\mathrm{C}=\mathrm{C}-$ $\mathrm{C}(\mathrm{O})-),{ }^{1} \mathrm{H}$ NMR $\left(\mathrm{CDCl}_{3}\right.$, TMS) (in ppm): $0.88\left(6 \mathrm{H}, \mathrm{CH}_{3} \mathrm{CH}_{2}-\right), 1.28\left(46 \mathrm{H}, \mathrm{CH}_{2}\right), 1.60$ (2H, $\left.\mathrm{CH}_{2}-\mathrm{CH}_{2} \mathrm{COO}\right), 2.10-2.40\left(4 \mathrm{H}, \mathrm{CH}_{2}-\right.$ $\left.\mathrm{CH}=, \mathrm{CH}_{2} \mathrm{COO}\right), 3.35\left(9 \mathrm{H},-\mathrm{N}^{+} \mathrm{CH}_{3}\right), 5.74$ $7.45(4 \mathrm{H}$, diene).

\section{Preparation of Polymerized Liposome}

DODPC $(0.2 \mathrm{~g})$ was dissolved in dehydrated chloroform and was slowly evaporated in a rotated sample tube to prepare thin lipid film on the inner surface of the tube. Distilled water $(20 \mathrm{ml})$ was added to the tube and a small unilamellar liposomes (SUV) suspension was prepared with sonicator (Tomy Seiko UR$200 \mathrm{P}$ ) at $60 \mathrm{~W}$ for $20 \mathrm{~min}$ under nitrogen atmosphere. The average radius of the liposomes was determined by TEM to be $14.0 \mathrm{~nm}$. THF solution of AIBN $\left(5 \mathrm{~mol}_{\%}^{\circ}\right.$ to the polymerizable lipids) was put into the liposome suspension. Nitrogen gas was bubbled through the suspension at a temperature above the $T_{\mathrm{c}}$ to incorporate AIBN into hydrophobic region of bilayer. The DODPC liposomes were polymerized at $60^{\circ} \mathrm{C}$. The polymerization conversion was analyzed by the decrease in the UV absorption intensity $\left(256.8 \mathrm{~nm}\right.$ at $\left.20^{\circ} \mathrm{C}\right)$ attributed to the diene groups.
Reconstitution of Liposomes from DODPC Polymers ${ }^{18}$

Small unilamellar liposomes prepared by sonication method were polymerized with AIBN at $60^{\circ} \mathrm{C}$ for $12 \mathrm{~h}$. Diene groups in 1-acyl chains were selectively polymerized by this procedure. ${ }^{11}$ The linear (comb like) DODPC polymers $\left(M_{n}=1.67 \times 10^{4}\right.$ by GPC calculated from polystyrene standard sample ${ }^{19}$ ) were obtained by dissolving the liposomes lyophilized from water into benzene. The lipid polymers were lyophilized again from benzene. The reconstitution of polymeric liposomes was performed by sonication of the polymerized DODPC powder. The average radius of the reconstituted liposomes was determined to be $16.2 \mathrm{~nm}$.

\section{$U V$-Spectroscopic Measurement of DODPC Liposomes}

An accurately diluted liposome suspension was put into a quartz cell. The absorption maximum $\left(\lambda_{\max }\right)$ of the diene groups was measured with UV spectrometry (Shimadzu MPS2000) under heating or cooling process with rate of $1^{\circ} \mathrm{C} \mathrm{min}^{-1}$.

\section{RESULTS AND DISCUSSION}

In this study, the phase transition behavior of two different polymerized liposomes was analyzed. One is that of acyl chains remained in the polymerized liposomes prepared by acyl chain-selective polymerization. . The other is that of lipids in outer or inner layer remained in the polymerized liposomes prepared by layer-selective polymerization. These are schematically indicated in Figure 1.

First, we introduced the excellent ability of DODPC lipid as probe molecules to examine the phase behavior.

\section{Phase Transition of Liposomes Analyzed with Diene-Containing Lipid}

Figure 2(a) shows the temperature dependence of wavelength at the absorption max- 

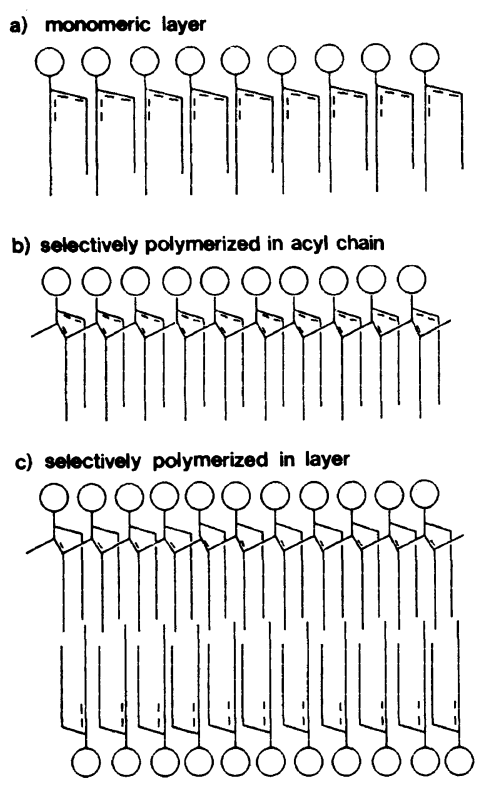

Figure 1. Schematic representation of selectively polymerized liposomes of diene-containing lipid.

imum $\left(\lambda_{\text {max }}\right)$ attributed to diene-groups of DODPC in liposomes. The $\lambda_{\max }$ for DODPC liposomes at liquid crystalline state was observed at $256.8 \mathrm{~nm}$. When the phase state of liposomes was changed to a gel state, it shifted to the shorter wavelength $(241.8 \mathrm{~nm})$. Because $\lambda_{\text {max }}$ of DODPC in $\mathrm{CHCl}_{3}$ was confirmed at $262.3 \mathrm{~nm}$ and did not show a significant change at any temperature, it was due to high interactions of diene-groups by the arrangement of diene-groups in gel state. ${ }^{17}$ Figure 2(b) shows the differential curve for the results of Figure 2(a). The peak of the curve corresponds to the phase transition temperature. DODPC was found to be an excellent probe molecule to detect not only the phase transition temperature of diene-containing lipid liposomes but also those of other lipid liposomes. ${ }^{17}$ Figure 3(a) shows phase transition behavior of saturated lipid liposomes detected by shift of the $\lambda_{\max }$ for DODPC. The incorporation amount of DODPC to the lipids was $5 \mathrm{~mol} \%$. In this case, the shift was considerably smaller than that of pure DODPC liposomes. When the lipid ratio

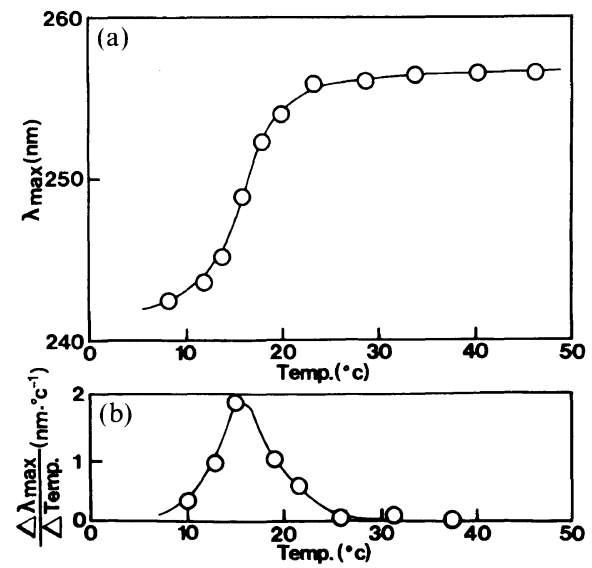

Figure 2. Temperature dependence of wavelength at the absorption maximum $\left(\lambda_{\max }\right)$ for diene groups of DODPC.
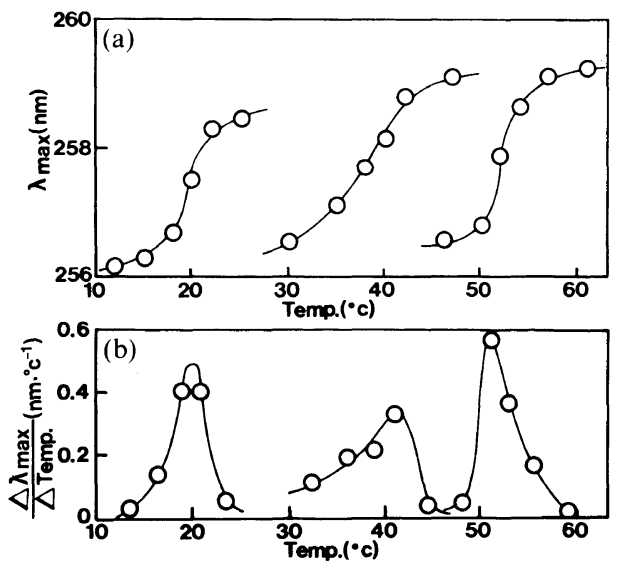

Figure 3. Temperature dependence of $\lambda_{\max }$ for diene groups of DODPC-saturated phospholipid $(1: 20$ by mol) mixed liposomes.

was increased, the magnitude of the shift and the peak in the differential curve approached those of pure DODPC liposomes. This indicates that the interaction of diene groups occurred due to the association of DODPC molecules in mixed membranes at temperatures below the $T_{\mathrm{c}}$ of the matrix lipids. The association disappeared when the temperature rose above the $T_{\mathrm{c}}$ and $\lambda_{\text {max }}$ was observed at the longest wavelength which meant no interaction of diene groups in the matrix lipids. The temperature for the maximum differential val- 
ue therefore indicated the phase transition temperature of the matrix (major) lipids only when the added amount of DODPC was small (generally less than $5 \mathrm{~mol} \%$ ). This method is based on the dispersion state of probe molecules, which is similar to the fluorescent measurement with DCPZn. The greatest advantage of the diene-type probe is that the probe molecule itself is a lipid and packed without disturbing the packing of other lipids. We could obtain direct information about the phase behavior of liposomes from the probe lipid.

\section{Phase Transition of Selectively Polymerized Liposomes}

The selective polymerization of dienegroups in 1- or 2-acyl chains of DODPC was achieved by using suitable radical initiators. If diene groups of either 1- or 2-acyl chains were selectively polymerized, the remaining diene groups were considered to be available to analyze the packing state of the acyl chains in polymeric liposomes. Figure 4 shows the temperature dependence of $\lambda_{\max }$ of diene groups in selectively polymerized liposomes with various polymerization conversions. In spite of the complete polymerization of diene groups of 1acyl chains (conversion: $50 \%$ ), the blue shift from $258.0 \mathrm{~nm}$ to $254.5 \mathrm{~nm}$ was apparently confirmed at $18^{\circ} \mathrm{C}$, which was a little higher than the main phase transition temperature of the monomeric DODPC liposomes $\left(16^{\circ} \mathrm{C}\right)$. We examined the phase transition behavior of the polymerized DODPC liposomes which could not be measured by other techniques such as NMR, DSC, ESR or fluorescence measurement.

The small $\lambda_{\text {max }}$ shift of polymerized DODPC liposomes was explained by the following experiments. The interactions between dienegroups of 1-acyl chains were considered to be different from those of 2-acyl chains because of the unsymmetrical packing of 1- and 2-acyl chains. Two kinds of diene-containing lipids were used here to prove this unequivalent

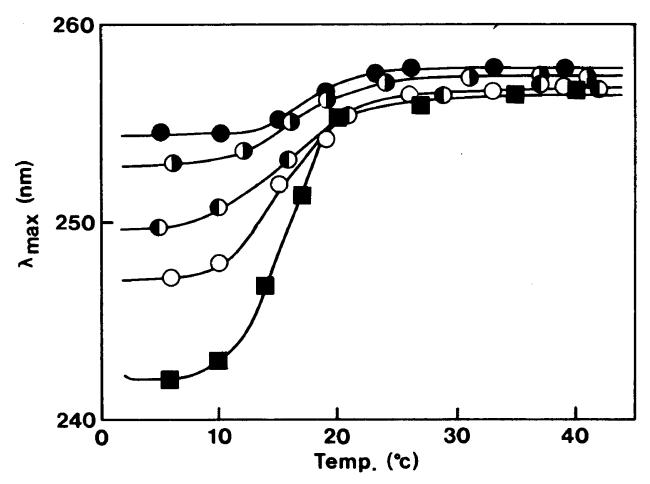

Figure 4. Temperature dependence of wavelength at the absorption maximum of polymerized DODPC liposomes with different polymerization conversion. $0 \%$ (口), $20.2 \%$ (○), $30.0 \%(\bigcirc), 43.9 \%(\bigcirc)$, and $51.0 \%$ (○).

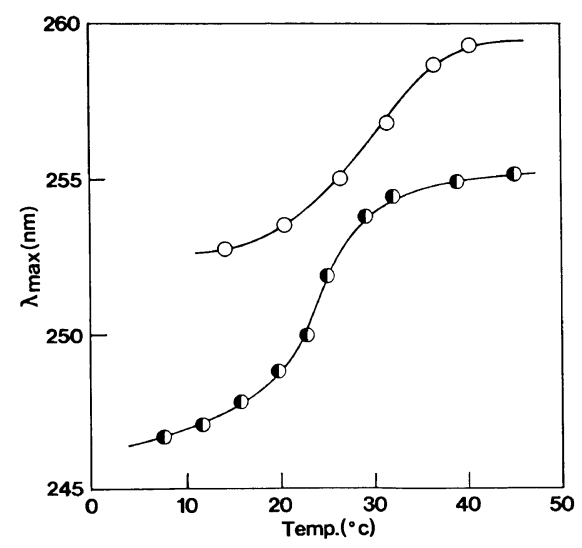

Figure 5. Temperature dependence of $\lambda_{\max }$ for diene groups of POPC $(O)$ and OPPC (O) liposomes.

interaction manner. One was 1-(2,4-octadecadienoyl)-2-palmitoyl-sn-glycero-3-phosphorylcholine (OPPC), which had one diene group in the 1-acyl chain. The other was 1palmitoyl-2-(2,4-octadecadienoyl)-sn-glycero-3-phosphorylcholine (POPC), which had one diene group in the 2-acyl chain. The temperature dependence of their $\lambda_{\max }$ is shown in Figure 5. The $\lambda_{\max }$ shift of POPC was very small compared with that of OPPC. The packing of diene groups of 2-acyl chains with large inclination to bilayer normal caused less interaction of the diene groups. When diene groups of 1 -acyl chains in DODPC liposomes 
were polymerized with AIBN, the interactions of the remained diene groups of the 2-acyl chains were therefore considered to be very small.

The $\lambda_{\max }$ shift of selectively polymerized DODPC was even smaller than that of POPC. This can be explained by the constrained packing of acyl chains bound to the polymer in bilayer. Polymerization of diene-containing lipid liposomes would form covalent $\mathrm{C}-\mathrm{C}$ bond between 1- and 4-position of diene group, ${ }^{20}$ and the orientation of diene moieties should be changed after the polymerization. The polymerization of diene groups of the 1-acyl chains of DODPC may induce considerable change in both the conformation of glycerol groups and the orientation of diene groups of the 2-acyl chains. Furthermore, polymerization may decrease the distance of adjacent acyl chains but increase that of the different polymers. ${ }^{21}$ The packing of acyl chains was concluded to be disturbed by the polymerization, which led to less interaction between diene groups on the average. The negative effect of polymerization on the lipid packing in liposome has been suggested without direct evidence. The present study is the first on the phase transition of polymerized lipids from $\lambda_{\max }$ shift of unreacted diene groups in the 2-acyl chains of DODPC polymers. The gel-to-liquid crystalline phase transition temperature of the DODPC polymers was a little higher than that of monomeric DODPC liposomes. The shift behavior of the phase transition temperature cannot be discussed simply because it may reflect the structural characteristics of both monomer and polymerized lipids. In case of polymerization of head groups, phase transition temperature detected by DSC shifted to a lower temperature. $^{9}$ Dioctadecadienoyl dimethyl ammonium bromide, which has the same acyl chains as DODPC, showed the phase transition temperature even after polymerization. $^{22}$ In this case, the phase transition temperature of polymerized liposomes was detected with DSC, densitometry and light- scattering measurement, and was confirmed to be a little higher than that of monomeric liposomes. In our case, polymerized DODPC liposomes showed less definite change from the gel to liquid crystalline state in comprison with monomeric liposomes because molecular packing and orientation of diene groups in gel state were disordered by polymer matrix and the mobility of acyl chains at liquid crystalline state was restricted by polymer chains. It should be noted that the average molecular weight and molecular weight distribution of the polymerized lipids were very important factors in phase behavior. ${ }^{19}$

\section{Phase Transition of Nonpolymerized Layer in Selectively Polymerized Liposomes}

We have already published that the preparation of liposomes whose half of bilayer was selectively polymerized. ${ }^{16} \mathrm{~A}$ water-soluble radical initiator (AAPD) works as a photosensitizer which generates radicals by irradiating $360 \mathrm{~nm}$ of UV-light independently of temperature. Since this initiator cannot penetrate the bilayer membrane at gel state, we can selectively polymerize an either layer of bilayer by incorporating the initiators in an either aqueous phase. The phase transition of these selectively polymerized liposomes in their layer should also be detected through $\lambda_{\text {max }}$ shift. For this, OPPC was used as a polymerizable lipid because polymerized DODPC had also dienegroups in 1-acyl chains which made the spectral analysis very complicated. Furthermore, OPPC had larger interaction compared with POPC as has already been shown in Figure 5. Figure 6 shows the phase transition behaviors of an inner- or an outer-layer of OPPC liposomes whose average radius was $40 \mathrm{~nm}$. Polymerization conversion of outer-layerpolymerized liposomes was $55 \%$ while that of inner-layer-polymerized liposomes remained $36 \%$. The liposomes which were polymerized unselectively until polymerization conversion of about $50 \%$ were also analyzed as a reference. Interestingly, $\lambda_{\max }$ of polymeric lipo- 


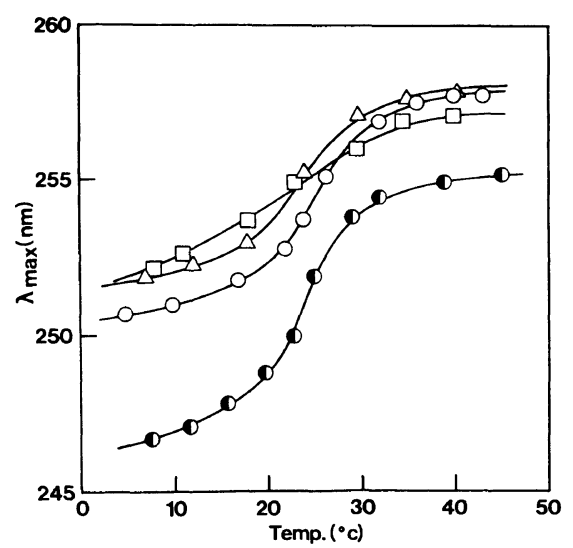

Figure 6. Phase transition of OPPC liposomes polymerized in outer $(O)$, inner $(\triangle)$, and both $(50 \%)(\square)$ layers. That of monomeric OPPC liposomes is shown as a reference (D).

somes shifted to longer wavelengths at any temperature in comparison with the curves for non-polymerized liposomes. This indicates that nonpolymerized region was also disturbed by the polymerization of the other layer. This was because different curvature and packing of polymerized region influenced the packing of nonpolymerized region. The cooperativity of phase transition of outer- or inner-polymerized liposomes was sharper than that of unselectively polymerized liposomes. This may be explained in terms of the size of the nonpolymerized region. Unselectively polymerized liposomes should be phase-separated into two phases; between polymerized and nonpolymerized region, the size of phase separated monomeric region was considerably smaller than that in the monomeric layer. It is interesting that $\lambda_{\text {max }}$ of inner-layer-polymerized liposomes were always observed in longer wavelength than those of outer-layer-polymerized liposomes. Thus, packing of the outer layer was more constrained than that of the inner layer.

\section{Analyses of Assembly State of Lipid Polymers}

DODPC whose 1-acyl chain was selectively polymerized by AIBN provided linear polymers which was soluble in organic solvent.

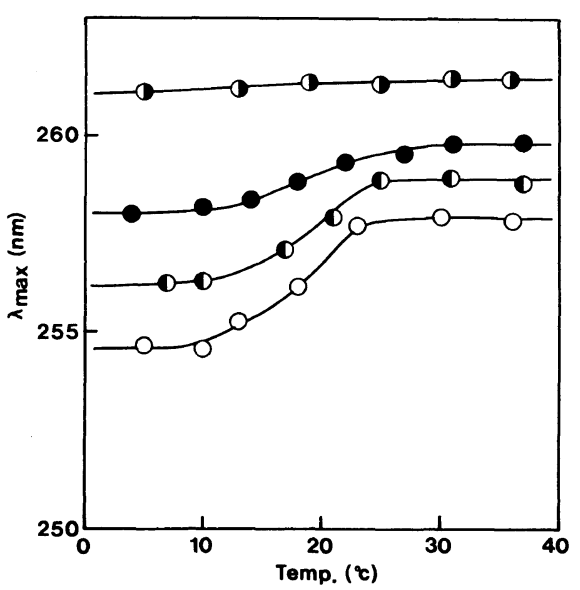

Figure 7. Temperature dependence of wavelength at the absorption maximum of polymerized DODPC liposome (O), reconstituted liposomes from DODPC polymers lyophilized from benzene solution $(O)$, redispersed liposomes from DODPC polymers lyophilized from liposome aqueous dispersion (D), and DODPC polymers in methanol (O).

The $\lambda_{\max }$ of unreacted diene groups in DODPC polymers was $262.7 \mathrm{~nm}$ in methanol, which meant no interaction of diene groups in methanol. There was no temperature dependence of DODPC polymers in methanol solution as shown in Figure 7. The DODPC polymers lyophilized from benzene solution therefore kept no liposome structure and afforded phospholipid polymers $\left(M_{n}=1.67 \times 10^{4}\right)$. These polymers were dispersed in water by sonication. Temperature dependence curve of the $\lambda_{\max }$ shifted to longer wavelength than the curve for monomeric but the phase transition was certainly confirmed at $18^{\circ} \mathrm{C}$. Thus diene groups started to interact with each other after the reconstitution of liposomes in water. The phase transition behavior of $\lambda_{\max }$ was however less sharp, and the curve for the temperature dependence was shifted to a longer wavelength than that in polymeric liposomes before reconstitution. This suggests that the acyl chain packing in the reconstituted polymeric liposomes was further disturbed because low segmental motion of phospholipid polymers prevented themselves from having 
the most stable acyl chain packing in assemblies. In case of DODPC polymers directly lyophilized from aqueous liposome dispersion, the liposome structure was kept. When these polymerized liposomes were redispersed in water by the extrusion method, ${ }^{18}$ the phase transition behavior of $\lambda_{\max }$ was similar to that of polymeric liposomes before lyophilization. $\lambda_{\max }$ shift behavior was significantly different in molecular packing among these 3 types of the polymerized liposomes. However, the difference has not hitherto been detected from CF release and fluorescence measurements. The incorporation of diene groups directly into acyl chains of phospholipid polymers was concluded to be very effective for analyzing the assembly of the lipid polymers.

\section{REFERENCES}

1. H. Ringsdorf, B. Schlarb, and J. Venzmer, Angew. Chem., Int. Ed. Engl., 27113 (1988).

2. G. Gregoriadis, Ed., "Liposome Technology," CRC Press, Boca Raton, Fl, 1984, and references therein.

3. J. Suurkuusk, B. R. Lenz, Y. Barenholz, R. L. Biltonen, and T. E. Thompson, Biochemistry, 15, 1393 (1976).

4. S. Takeoka, H. Ohno, N. Hayashi, and E. Tsuchida, J. Controlled Release (1989) (in press).
5. L. Gros, H. Ringsdorf, and H. Schupp, Angew. Chem., Int. Ed. Engl., 20, 305 (1981).

6. H. Ohno, S. Takeoka, N. Hayashi, and E. Tsuchida, Makromol. Chem., Rapid Commun., 8, 215 (1987).

7. H. Ohno, S. Takeoka, and E. Tsuchida, Polym. Bull., 14, 487 (1985).

8. H. Gaub, E. Sackmann, R. Büschl, and $\mathbf{H}$. Ringsdorf, Biophys. J., 45, 725 (1984).

9. R. Büschl, T. Folda, and H. Ringsdorf, Makromol. Chem. Suppl., 6, 245 (1984).

10. L. Wang, E. Shoji, H. Ohno, and E. Tsuchida, Polym. Bull., 21, 195 (1989).

11. H. Ohno, Y. Ogata, and E. Tsuchida, Macromolecules, 20, 929 (1987).

12. H. Ohno, S. Takeoka, and E. Tsuchida, Bull. Chem. Soc. Jpn., 60, 2945 (1987).

13. H. Ohno, S. Takeoka, H. Iwai, and E. Tsuchida, J. Polym. Sci., Polym. Chem. Ed., 25, 2737 (1987).

14. S. Takeoka, E. Hasegawa, H. Ohno, and E. Tsuchida, Bull. Chem. Soc. Jpn., 61, 2007 (1988).

15. H. Ohno, S. Takeoka, H. Iwai, and E. Tsuchida, Macromolecules, 21, 319 (1988).

16. H. Ohno, S. Takeoka, H. Iwai, and E. Tsuchida, Macromolecules, 22, 61 (1989).

17. S. Takeoka, H. Ohno, H. Iwai, and E. Tsuchida, Bull. Chem. Soc. Jpn., 62, 102 (1989).

18. S. Takeoka, H. Ohno, H. Iwai, and E. Tsuchida, $J$. Polym. Sci., Polym. Chem. Ed., (in contribution).

19. S. Takeoka, N. Kimura, H. Ohno and E. Tsuchida, Polym. Prepr., Jpn., 37, 478 (1988).

20. A. Laschewsky and H. Ringsdorf, Macromolecules, 21, (1988).

21. W. F. Reed, Macromolecules, 18, 2402 (1985).

22. H. Gaub, R. Buschl, H. Ringsdorf, and E. Sackmann, Chem. Phys. Lipids, 37, 19 (1985). 